\title{
INHOMOGENEOUS FLUIDS IN AN EXTERNAL FIELD
}

\author{
F. LADO \\ Department of Physics \\ North Carolina State University \\ Raleigh, NC, 27695-8202, USA \\ AND \\ E. LOMBA \\ Instituto de Química Física Rocasolano, CSIC \\ Serrano 119 \\ 28006 Madrid, Spain
}

\section{Introduction}

The Gibbsian $N$-body density function of a Hamiltonian $H_{N}$ that is rotationally and translationally invariant must itself be rotationally and translationally invariant, as must then also be all reduced $n$-body density functions of this Hamiltonian. In particular, the one-body density is a constant and the two-body density depends only on relative coordinates. An external field destroys this homogeneity, producing anisotropy or nonuniformity [1, 2], and so makes necessary the joint calculation of the coupled one-body and two-body density functions. A striking if familiar example of the response of a bulk system to an external field is ferromagnetism. We shall use this particular case here to present a general procedure to compute the coupled one-body and two-body density functions of an inhomogeneous classical fluid in an external field. Remarkably, the procedure is no more difficult to carry through than similar calculations for ordinary homogeneous systems.

Perhaps the simplest model of a disordered continuum system exhibiting ferromagnetic behavior is a fluid of hard spheres with embedded Heisenberg spins described using classical statistical mechanics $[3,4,5,6,7]$. On one hand, the simplifications of this model compared to magnetic dipole-dipole interactions are significant for simulation. The dipole-dipole model presents

substantial conceptual difficulties in connection with the nonexistence of the thermodynamic limit for orientationally ordered phases. Moreover, its 
simulation results are strongly dependent on the boundary conditions. Simulations for the Heisenberg spin model are free of these problems. (The integral equation formalism developed below serves equally well for either potential.) On the other hand, disordered Heisenberg spin systems are of interest in their own right. It has been known for some time that $\mathrm{Co} / \mathrm{P}$ alloys [8] and $\mathrm{Co} / \mathrm{Au}$ melts [9] have a tendency to form amorphous ferromagnets, although their "true liquid" nature has been questioned because of the technical difficulties posed by undercooling below the Curie temperature. While these systems might be more representative of quenched spin fluids, very recently Albrecht and coworkers [10] have finally managed to undercool a $\mathrm{Co}_{80} \mathrm{Pd}_{20}$ melt below its Curie temperature at zero field, thus obtaining the first evidence of ferromagnetic behavior in a liquid metal under conditions where the Heisenberg exchange interaction absolutely dominates the magnetic dipole-dipole contribution.

The Heisenberg spin fluid in an external magnetic field $\mathbf{B}_{0}$ is defined by the canonical partition function $Z=Z^{i d} Z^{e x}$, where

$$
\begin{aligned}
Z^{i d}= & \frac{1}{N ! \Lambda^{3 N}} \int \prod_{j=1}^{N}\left[d \mathbf{r}_{j} d \omega_{j}\right] \exp \left(\beta \sum_{j} \boldsymbol{\mu}_{j} \cdot \mathbf{B}_{0}\right) \\
= & \frac{1}{N !}\left(\frac{V}{\Lambda^{3}}\right)^{N}\left[4 \pi \frac{\sinh \beta \mu B_{0}}{\beta \mu B_{0}}\right]^{N}, \\
Z^{e x}= & \frac{1}{(4 \pi V)^{N}} \int \prod_{j=1}^{N}\left[d \mathbf{r}_{j} d \omega_{j} f_{0}\left(\omega_{j}\right)\right] \\
& \times\left(-\beta \sum_{i<j} u_{0}\left(r_{i j}\right)-\beta \sum_{i<j} u_{s s}\left(r_{i j}, \omega_{i}, \omega_{j}\right)\right) .
\end{aligned}
$$

Here $\beta=1 / k_{B} T$ is the inverse Kelvin temperature, with $k_{B}$ Boltzmann's constant, $\Lambda$ the de Broglie thermal wavelength, and $\omega=(\theta, \phi)$ the orientation of the magnetic dipole moment $\boldsymbol{\mu}$, referred to the uniform field $\mathbf{B}_{0}$ as the $z$ direction. The interaction energies are written as pairwise sums of the hard sphere potential $u_{0}(r)$ for spheres of diameter $\sigma$ and the Heisenberg spin-spin potential $u_{s s}\left(r, \omega_{1}, \omega_{2}\right)$ for $r>\sigma$,

$$
u_{s s}\left(r, \omega_{1}, \omega_{2}\right)=-J(r) \hat{\mathbf{s}}_{1} \cdot \hat{\mathbf{s}}_{2}, \quad \beta J(r)=K \frac{e^{-\kappa(r / \sigma-1)}}{r / \sigma},
$$

where $\hat{\mathbf{s}}$ is the unit spin vector in the direction of $\boldsymbol{\mu}$. In this last expression, the dimensionless coupling strength $K$ may be read as the inverse reduced temperature, $K=1 / T^{*}$, while $\kappa$ is a dimensionless range parameter. Finally, in Eq. (2), $f_{0}(\omega)$ is the normalized one-body orientational distribution 
of a noninteracting spin system,

$$
f_{0}(\omega)=\frac{e^{\beta \mu B_{0} \cos \theta}}{\sinh \left(\beta \mu B_{0}\right) / \beta \mu B_{0}} .
$$

\section{Integral equation formulation}

The key quantities needed for a complete magnetic and thermodynamic description of this system are the one-body and two-body density functions,

$$
\begin{aligned}
\rho^{(1)}(\mathbf{r}, \omega) & =\left\langle\sum_{j=1}^{N} \delta\left(\mathbf{r}-\mathbf{r}_{j}\right) \delta\left(\omega-\omega_{j}\right)\right\rangle=\frac{\rho}{4 \pi} f(\omega), \\
\rho^{(2)}\left(\mathbf{r}, \omega, \mathbf{r}^{\prime}, \omega^{\prime}\right) & =\left\langle\sum_{i \neq j} \delta\left(\mathbf{r}-\mathbf{r}_{i}\right) \delta\left(\omega-\omega_{i}\right) \delta\left(\mathbf{r}^{\prime}-\mathbf{r}_{j}\right) \delta\left(\omega^{\prime}-\omega_{j}\right)\right\rangle \\
& =\frac{\rho^{2}}{(4 \pi)^{2}} f(\omega) f\left(\omega^{\prime}\right) g\left(\left|\mathbf{r}-\mathbf{r}^{\prime}\right|, \omega, \omega^{\prime}\right),
\end{aligned}
$$

where $\rho=N / V$ is the density and $f(\omega)$ the one-body orientational distribution in the interacting fluid. Equation (6) defines the generalized pair distribution function $g\left(r, \omega, \omega^{\prime}\right)$ of the inhomogeneous spin system in an external magnetic field. The angular brackets in these definitions denote a canonical ensemble average with the Hamiltonian of $Z$ above.

The basic equations that determine the distribution functions $f(\omega)$ and $g\left(r, \omega, \omega^{\prime}\right)$ are well known $[11,12]$. The one-body density can be differentiated with respect to $x=\cos \theta$ to give

$$
\frac{d}{d x} \ln \left[\frac{f(\omega)}{f_{0}(\omega)}\right]=-\frac{\rho}{4 \pi} \int d \mathbf{r} d \omega^{\prime} f\left(\omega^{\prime}\right) g\left(r, \omega, \omega^{\prime}\right) \frac{d \beta u_{s s}\left(r, \omega, \omega^{\prime}\right)}{d x},
$$

the first member of a Kirkwood-Born-Green-Yvon hierarchy. Calculation of $f(\omega)$ from this equation requires knowing $g$. In classical liquid state theory, the pair distribution function is obtained from the Ornstein-Zernike (OZ) equation and a closure relation $[11,12]$. The first of these, generalized for anisotropy, reads

$$
\begin{aligned}
\gamma\left(r_{12}, \omega_{1}, \omega_{2}\right)= & \frac{\rho}{4 \pi} \int d \mathbf{r}_{3} d \omega_{3} f\left(\omega_{3}\right) \\
& \times\left[\gamma\left(r_{13}, \omega_{1}, \omega_{3}\right)+c\left(r_{13}, \omega_{1}, \omega_{3}\right)\right] c\left(r_{32}, \omega_{3}, \omega_{2}\right)
\end{aligned}
$$

for the indirect correlation function $\gamma=g-1-c$, where $c$ is the direct correlation function. The second, or closure, relation expresses $c$ back in 
terms of $\gamma$ and the model's pair interactions,

$$
\begin{aligned}
c\left(r, \omega_{1}, \omega_{2}\right)= & \exp \left[-\beta u_{0}(r)-\beta u_{s s}\left(r, \omega_{1}, \omega_{2}\right)+\gamma\left(r, \omega_{1}, \omega_{2}\right)\right. \\
& \left.+b\left(r, \omega_{1}, \omega_{2}\right)\right]-1-\gamma\left(r, \omega_{1}, \omega_{2}\right) .
\end{aligned}
$$

This relation must be supplemented with an approximation for $b$, the socalled bridge function.

We have recently described $[13,14]$ a practical approach to solving Eqs. (7)-(9) for any classical fluid in an external field using the same numerical procedures already employed for homogeneous systems. The essential ingredient turns out to be orthogonal polynomials tailored to the specific one-body distribution of the fluid [15]. The usual procedure for orientationdependent functions such as $\gamma\left(r, \omega_{1}, \omega_{2}\right)$ is to expand in spherical harmonics $Y_{l m}(\omega)[16]$,

$$
\begin{aligned}
\gamma\left(r, \omega_{1}, \omega_{2}\right) & =4 \pi \sum_{l_{1}, l_{2}, m} \gamma_{l_{1} l_{2} m}(r) Y_{l_{1} m}\left(\omega_{1}\right) Y_{l_{2} \bar{m}}\left(\omega_{2}\right), \\
Y_{l m}(\omega) & =\frac{1}{\sqrt{4 \pi}}(-1)^{m} e^{i m \phi} P_{l m}(\cos \theta),
\end{aligned}
$$

where $P_{l m}(\cos \theta)$ is the familiar associated Legendre function, but here renormalized to two, and $\bar{m}=-m$. This expansion in fact makes the solution of the inhomogeneous liquid equations very difficult. We will instead expand in modified spherical harmonics,

$$
\begin{aligned}
\gamma\left(r, \omega_{1}, \omega_{2}\right) & =4 \pi \sum_{l_{1}, l_{2}, m} \gamma_{l_{1} l_{2} m}(r) \mathcal{Y}_{l_{1} m}\left(\omega_{1}\right) \mathcal{Y}_{l_{2} \bar{m}}\left(\omega_{2}\right), \\
\mathcal{Y}_{l m}(\omega) & =\frac{1}{\sqrt{4 \pi}}(-1)^{m} e^{i m \phi} \mathcal{P}_{l m}(\cos \theta) .
\end{aligned}
$$

The modified Legendre functions $\mathcal{P}_{l m}(\cos \theta)$ are explicitly constructed, beginning with $\mathcal{P}_{00}(\cos \theta)=1$, using the Gram-Schmidt method $[17,18]$ with the orthonormality condition

$$
\frac{1}{2} \int_{-1}^{1} d x f(x) \mathcal{P}_{l m}(x) \mathcal{P}_{l^{\prime} m}(x)=\delta_{l l^{\prime}},
$$

where $f(\cos \theta)$ is the one-body distribution of the fluid. For an isotropic fluid with $f(x)=1$, this construction produces the standard Legendre functions $P_{l m}(x)$ (normalized to two). For an anisotropic fluid with $f(x) \neq 1$, the difference between $P_{l m}(x)$ and $\mathcal{P}_{l m}(x)$ is crucial.

The numerical evaluation of the OZ equation is simpler in Fourier transform representation, which deconvolutes the $\mathbf{r}$ integral. Thus, in transform space, Eq. (8) becomes

$$
\tilde{\gamma}\left(k, \omega_{1}, \omega_{2}\right)=\frac{\rho}{4 \pi} \int d \omega_{3} f\left(\omega_{3}\right)\left[\tilde{\gamma}\left(k, \omega_{1}, \omega_{3}\right)+\tilde{c}\left(k, \omega_{1}, \omega_{3}\right)\right] \tilde{c}\left(k, \omega_{3}, \omega_{2}\right),
$$


with one remaining integration. This final integral can also be eliminated and the evaluation of the OZ equation reduced to algebra by now expanding the pair functions as in (12); Eq. (15) then becomes

$$
\tilde{\gamma}_{l_{1} l_{2} m}(k)=(-1)^{m} \rho \sum_{l_{3}}\left[\tilde{\gamma}_{l_{1} l_{3} m}(k)+\tilde{c}_{l_{1} l_{3} m}(k)\right] \tilde{c}_{l_{3} l_{2} m}(k) .
$$

The significant feature here is that this OZ equation for an inhomogeneous fluid in an external field is identical to that of an ordinary homogeneous fluid [16] and so, along with some closure equation familiar from simple liquids, can be solved for $g\left(r, \omega_{1}, \omega_{2}\right)$ with the same algorithms already used for homogeneous systems $[19,20]$.

Equations (7)-(9) being nonlinear, their solution is iterative. Given a set of approximate coefficients $\gamma_{l_{1} l_{2} m}(r)$, an improved set is produced by the following steps:

1. Closure: $\gamma_{l_{1} l_{2} m}(r) \rightarrow c_{l_{1} l_{2} m}(r)$.

$$
\begin{aligned}
\gamma\left(r, x_{1}, x_{2}, y\right)= & \sum_{l_{1}, l_{2}, m} \gamma_{l_{1} l_{2} m}(r) \mathcal{P}_{l_{1} m}\left(x_{1}\right) \mathcal{P}_{l_{2} m}\left(x_{2}\right)(-1)^{m} T_{m}(y) \\
c\left(r, x_{1}, x_{2}, y\right)= & \exp \left[-\beta u_{0}(r)-\beta u_{s s}\left(r, x_{1}, x_{2}, y\right)+\gamma\left(r, x_{1}, x_{2}, y\right)\right. \\
& \left.+b\left(r, x_{1}, x_{2}, y\right)\right]-1-\gamma\left(r, x_{1}, x_{2}, y\right) \\
c_{l_{1} l_{2} m}(r)= & \frac{1}{4 \pi} \int_{-1}^{1} \frac{d y}{\sqrt{1-y^{2}}} \int_{-1}^{1} d x_{2} \int_{-1}^{1} d x_{1} f\left(x_{1}\right) f\left(x_{2}\right) \\
& \times c\left(r, x_{1}, x_{2}, y\right) \mathcal{P}_{l_{1} m}\left(x_{1}\right) \mathcal{P}_{l_{2} m}\left(x_{2}\right)(-1)^{m} T_{m}(y)
\end{aligned}
$$

2. Transform: $c_{l_{1} l_{2} m}(r) \rightarrow \tilde{c}_{l_{1} l_{2} m}(k)$.

$$
\tilde{c}_{l_{1} l_{2} m}(k)=\frac{4 \pi}{k} \int_{0}^{\infty} d r r c_{l_{1} l_{2} m}(r) \sin k r
$$

3. OZ equation: $\tilde{c}_{l_{1} l_{2} m}(k) \rightarrow \tilde{\gamma}_{l_{1} l_{2} m}(k)$.

$$
\tilde{\Gamma}_{m}(k)=(-1)^{m} \rho \tilde{C}_{m}(k) \tilde{C}_{m}(k)\left[I-(-1)^{m} \rho \tilde{C}_{m}(k)\right]^{-1}
$$

4. Inverse transform: $\tilde{\gamma}_{l_{1} l_{2} m}(k) \rightarrow \gamma_{l_{1} l_{2} m}(r)$.

$$
\gamma_{l_{1} l_{2} m}(r)=\frac{1}{2 \pi^{2} r} \int_{0}^{\infty} d k k \tilde{\gamma}_{l_{1} l_{2} m}(k) \sin k r
$$


In these equations, $x=\cos \theta, y=\cos \phi$, and $T_{m}(y)$ is a Chebyshev polynomial; further, in Eq. (21), $I$ is the unit matrix while $\tilde{\Gamma}_{m}(k)$ and $\tilde{C}_{m}(k)$ are symmetric matrices with elements $\tilde{\gamma}_{l_{1} l_{2} m}(k)$ and $\tilde{c}_{l_{1} l_{2} m}(k)$, respectively, with $l_{1}, l_{2} \geq m$. The inversion (19) is performed using $n$-point Gaussian quadratures with the zeroes of $\mathcal{P}_{n 0}(x)$ and $T_{n}(y)$. Upon convergence of the $\gamma_{l_{1} l_{2} m}(r)$ coefficients, the generalized pair distribution function $g$ is finally computed as

$$
g\left(r, \omega_{1}, \omega_{2}\right)=\exp \left[-\beta u_{0}(r)-\beta u_{s s}\left(r, \omega_{1}, \omega_{2}\right)+\gamma\left(r, \omega_{1}, \omega_{2}\right)+b\left(r, \omega_{1}, \omega_{2}\right)\right] .
$$

This solution for $g\left(r, \omega_{1}, \omega_{2}\right)$ is obtained using the polynomials $\mathcal{P}_{l m}(x)$ generated with the current $f(x)$. One now returns to Eq. (7) and updates the one-body distribution $f(x)$. In expanded form, this equation is

$$
\begin{gathered}
\frac{d}{d x} \ln \left[\frac{f(x)}{f_{0}(x)}\right]=\sum_{l_{1}, l_{2}, m} \xi_{l_{1} l_{2} m} \mathcal{P}_{l_{1} m}(x) \frac{d \mathcal{P}_{l_{2} m}(x)}{d x}, \\
\xi_{l_{1} l_{2} m}=-\rho \int d \mathbf{r} \sum_{l_{3}} g_{l_{1} l_{3} m}(r) u_{l_{3} l_{2} \bar{m}}(r),
\end{gathered}
$$

where the $u_{l_{1} l_{2} m}(r)$ are the (known) coefficients of the spin-spin interaction $u_{s s}\left(r, \omega_{1}, \omega_{2}\right)$, so that finally

$$
\ln f(x)=\ln f_{0}(x)+\sum_{l=0}^{\infty} a_{l} \mathcal{P}_{l 0}(x) .
$$

Here $a_{l}$ for $l>0$ is determined by numerical (Gaussian) integration of Eq. (23) and $a_{0}$ by normalization. The $n$-point Gaussian quadrature is exact for polynomial integrands of degree less than $2 n$, a condition that always holds for the computed $a_{l}$ coefficients. For the calculations reported in Ref. [14], it turns out that the term linear in $x$ dominates the series expansion in Eq. (25), so that an effective field $B$ acting on a particle that includes the magnetic effects of the particle's surroundings through the coefficient $a_{1}$ can be calculated from this equation. In the absence of an interaction $\left(a_{1}=0\right)$ this field is of course just $B_{0}$.

The iterations for $f(\omega)$ and $g\left(r, \omega_{1}, \omega_{2}\right)$ are continued until both functions are self-consistently determined. The only approximation in the calculation is the unavoidable one in every liquid state calculation to date, the representation of the bridge function $b\left(r, \omega_{1}, \omega_{2}\right)$ [11].

The angular projection coefficients of Eq. (12) for, say, $g\left(r, \omega_{1}, \omega_{2}\right)$ cannot be directly compared with the standard projections typically calculated in a simulation, since these correspond to the usual spherical harmonic basis. However, it is straightforward to recover one set of coefficients from the 
other given the moments of the one-particle distribution function $f(x)$. For example, the (unnormalized) projection of $g\left(r, \omega_{1}, \omega_{2}\right)$ onto the rotational invariant $\hat{\mathbf{s}}_{1} \cdot \hat{\mathbf{s}}_{2}$ is found to be

$$
\begin{aligned}
h^{110}(r)= & 3\left\{\langle x\rangle^{2} g_{000}(r)+\sigma_{x}\langle x\rangle\left[g_{100}(r)+g_{010}(r)\right]\right. \\
& \left.+\sigma_{x}^{2} g_{110}(r)-\left[1-\left\langle x^{2}\right\rangle\right] g_{111}(r)\right\} .
\end{aligned}
$$

For an isotropic system, with $\langle x\rangle=0$ and $\left\langle x^{2}\right\rangle=1 / 3$, this reduces to the usual $h^{110}(r)=g_{110}(r)-2 g_{111}(r)$.

We can further compute the complete magnetic and thermodynamic properties of a Heisenberg spin fluid in a uniform magnetic field $\mathbf{B}_{0}$. The net magnetization in the direction $\alpha=x, y, z$ is

$$
M_{\alpha}=\mu\left\langle\sum_{j} s_{j \alpha}\right\rangle=\frac{1}{\beta} \frac{\partial \ln Z}{\partial B_{0 \alpha}},
$$

and so the magnetic susceptibility components are

$$
\begin{aligned}
\chi_{\alpha \beta} \equiv & \frac{1}{V} \frac{\partial M_{\alpha}}{\partial B_{0 \beta}}=\rho \beta \mu^{2}\left\{\left\langle s_{1 \alpha} s_{1 \beta}\right\rangle-\left\langle s_{1 \alpha}\right\rangle\left\langle s_{1 \beta}\right\rangle\right. \\
& \left.+(N-1)\left[\left\langle s_{1 \alpha} s_{2 \beta}\right\rangle-\left\langle s_{1 \alpha}\right\rangle\left\langle s_{2 \beta}\right\rangle\right]\right\} .
\end{aligned}
$$

Only the diagonal elements are nonvanishing. With $\mathbf{B}_{0}$ defining the $z$ direction, we get the longitudinal and transverse magnetic susceptibilities as

$$
\begin{aligned}
\chi_{z z} / \rho \beta \mu^{2} & =\sigma_{x}^{2}\left[1+\rho \tilde{h}_{110}(0)\right]+\sigma_{x}\langle x\rangle\left[\rho \tilde{h}_{100}(0)+\rho \tilde{h}_{010}(0)\right] \\
\chi_{y y} / \rho \beta \mu^{2} & =\frac{1}{2}\left(1-\left\langle x^{2}\right\rangle\right)\left[1-\rho \tilde{h}_{111}(0)\right] .
\end{aligned}
$$

For an isotropic system, these susceptibility expressions reduce to familiar forms.

The internal energy $U$, pressure $p$, and isothermal compressibility $K_{T}$ are calculated with the standard formulas of homogeneous fluids [11, 16], again thanks to the special orthogonality of the $\mathcal{Y}_{l m}(\omega)$.Finally, an expression for the Helmholtz free energy $A$ is given in Ref. [14].

\section{Computed results}

We have obtained the distribution functions $f(\omega)$ and $g\left(r, \omega_{1}, \omega_{2}\right)$ for the Heisenberg spin fluid in a magnetic field $\mathbf{B}_{0}$ using a reference version of the Zerah-Hansen (RZH) closure [21]; for $r>\sigma$, this is

$$
\begin{aligned}
c^{R Z H}\left(r, \omega_{1}, \omega_{2}\right)= & \left\{\operatorname { e x p } \left(m ( r ) \left[-\beta u_{s s}\left(r, \omega_{1}, \omega_{2}\right)+\gamma\left(r, \omega_{1}, \omega_{2}\right)\right.\right.\right. \\
& \left.\left.\left.+b_{\mathrm{HS}}(r ; \sigma)\right]\right)-1\right\} / m(r)-\gamma\left(r, \omega_{1}, \omega_{2}\right) .
\end{aligned}
$$


The exact relation $c\left(r, \omega_{1}, \omega_{2}\right)=-1-\gamma\left(r, \omega_{1}, \omega_{2}\right)$ holds for $r<\sigma$.

The RZH closure features a mixing function $m(r)=1-\exp (-\alpha r)$ with a parameter $\alpha$ that is fixed by requiring consistency between the virial and compressibility bulk moduli. (However, see below for the case $B_{0} \rightarrow 0$.) The reference hard sphere bridge function $b_{\mathrm{HS}}(r ; \sigma)$, added by us in Eq. (31) to the usual ZH closure [21], ensures the RZH closure yields good hard sphere results in the limit of small $K$ and improves the thermodynamic results overall.

To be consistent with earlier work $[4,5]$, we have truncated the spinspin interaction at $r=r_{c} \equiv 2.5 \sigma$; this hardly affects the thermodynamics (aside from the pressure) but does considerably raise the critical $K$ value. (Some results for the untruncated potential obtained using density functional theory and Monte Carlo simulation can be found in Refs. [6] and [7].)

We report here calculations for $\kappa=1, \rho \sigma^{3}=0.7, K=1 / T^{*}=0$ to 0.5 , and two values of the external field, $\beta \mu B_{0}=1$ and 0 , using the RZH closure. As noted earlier, we find for these cases that the one-body orientational distribution function $f(x)$ continues to be well described by the functional form of $f_{0}(x)$, but with an effective field $B$,

$$
f(x)=\frac{e^{\beta \mu B x}}{\sinh (\beta \mu B) / \beta \mu B} .
$$

In the limit $B_{0} \rightarrow 0$, the effective field $B$ is zero for $K<K_{c}$ and finite for $K>K_{c}$, where $K_{c}=1 / T_{c}^{*}$ is then the computed Curie point. This zero field limit has recently been the focus of several works $[4,5,6,7]$, but until now no theoretical approximation has been able to completely describe the ordered phase having spontaneous magnetization.

For $K>K_{c}$, there is a singularity when $B_{0} \rightarrow 0$ that is different from the second order paramagnetic-ferromagnetic transition at $K=K_{c}$. In fact, the $B_{0}=0$ line corresponds in the $B_{0}-M$ plane (where $M$ is the magnetization per particle) to the spinodal line that indicates the equilibrium between phases with positive and negative magnetization. Consequently, the transverse susceptibility $\chi_{y y}$ (but not the longitudinal component $\chi_{z z}$ ) will diverge [22] as $B_{0} \rightarrow 0$, reflecting the negligible cost of rotating an ordered sample in the absence of an external field. It turns out that the optimization of $\alpha$ in (31) to achieve thermodynamic consistency leads to a $\chi_{y y}$ that diverges at small but non-zero fields. This deficiency is easily cured if one relaxes the thermodynamic consistency requirement and instead optimizes the $\alpha$ parameter by displacing the divergence of $\chi_{y y}$ to the $B_{0}=0$ line. We find that the thermodynamics is hardly affected by the new choice of $\alpha$. 


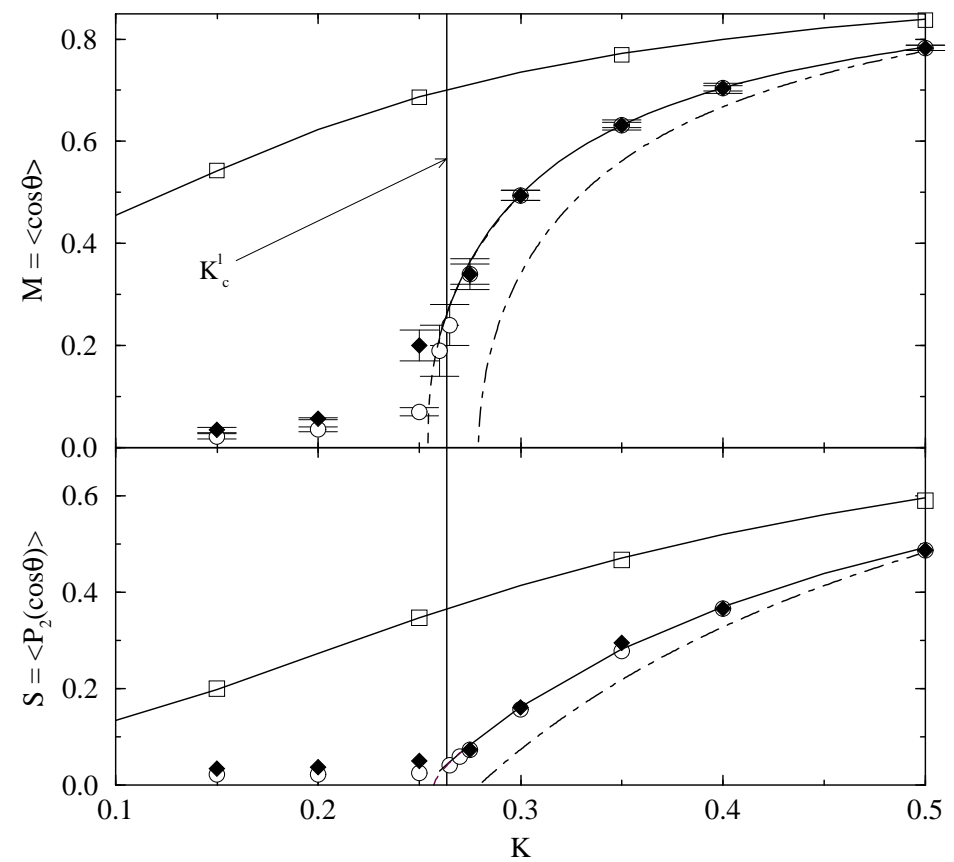

Figure 1. Magnetization per particle $M$ and second order parameter $S$ as functions of inverse temperature $K=1 / T^{*}$ obtained from MC simulation [squares for $\beta \mu B_{0}=1$ and white circles (2048 particle sample) and black diamonds (1372 particle sample) for $\left.\beta \mu B_{0}=0\right]$. The RZH integral equation results appear as solid lines. The dashed lines correspond to a power law fit to the RZH data just above $K_{c}^{u}$. The dash-dot curves represent density functional theory estimates [7].

Using Monte Carlo simulation, Nijmeijer and Weis [4] found that the paramagnetic-ferromagnetic transition for $\rho \sigma^{3}=0.7$ with the truncated potential occurs at $K_{c}=0.264 \pm 0.001$. Our calculation in the paramagnetic phase and zero field limit yields a divergence in $\chi_{z z}$ at $K_{c}{ }^{l}=0.2645$, in excellent agreement with the simulation value.

In Fig. 1 we show the values of the magnetization per particle $M \equiv$ $M_{z}=\langle\cos \theta\rangle$ and the second order parameter $S=\left\langle P_{2}(\cos \theta)\right\rangle$ obtained from the theory and from a standard NVT Monte Carlo simulation using 864 (for $B_{0}=1$ ), 1372 and 2048 particles (for $B_{0}=0$ ), and averages over 40000 configurations. Size effects are noticeable for $B_{0}=0$ close to and below (i.e., $K<K_{c}$ ) the critical point. We include in Fig. 1 some zero-field results from density functional theory in the modified mean field approximation [7]. In the vicinity of the critical temperature, one encounters convergence difficulties in solving the integral equation as spin-spin correlations become long ranged. (Both $\chi_{z z}$ and $\chi_{y y}$ in Eqs. (29) and (30) diverge, with $\chi_{z z}$ exhibiting the characteristic $\lambda$-type divergence of second- 


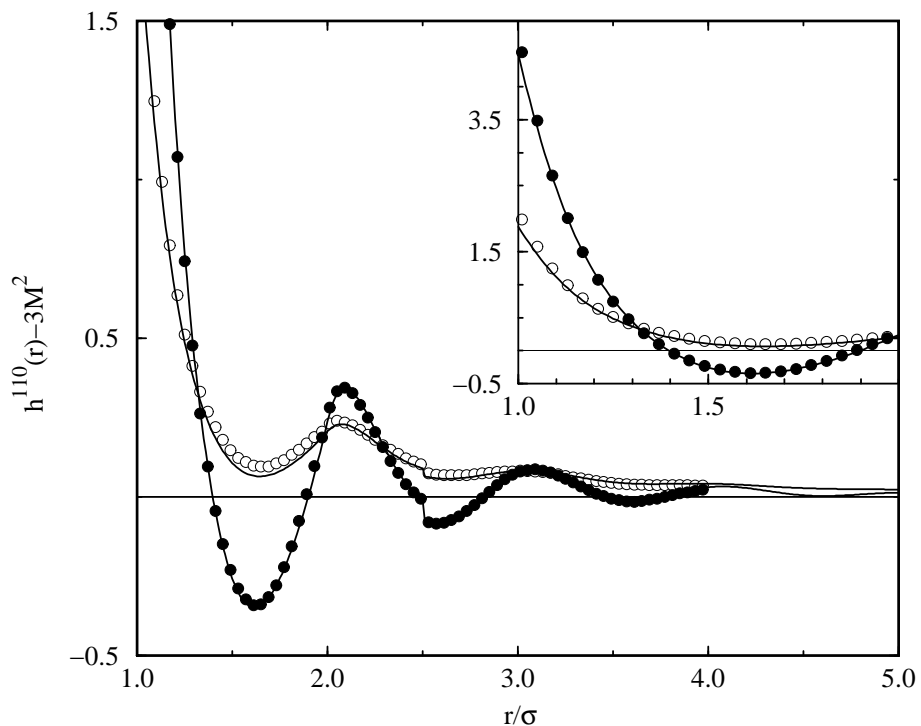

Figure 2. Behavior of the angular correlation function $h^{110}(r)$ as the Curie temperature is approached in the ferromagnetic phase at zero field obtained from MC simulation using 1372 particles (white circles correspond to $K=0.275$ and black circles to $K=0.5$ ) for a density $\rho \sigma^{3}=0.7$. The RZH results are shown as solid lines in both cases. The long-range limiting value $3 M^{2}$ has been subtracted from both theory and simulation results to facilitate the comparison between the two temperatures. The discontinuity at $r=2.5 \sigma$ is due to the potential truncation.

order transitions.) In Fig. 2 we illustrate the increase in the range of the relevant angular correlation function, $h^{110}(r)$, as the Curie temperature is approached. The function $h^{110}(r)$, defined in Eq. (26), describes most of the orientational behavior of the spin fluid, since it is the projection of $g\left(r, \omega_{1}, \omega_{2}\right)$ onto the rotational invariant $\hat{\mathbf{s}}_{1} \cdot \hat{\mathbf{s}}_{2}=\cos \theta_{12}$. From Eq. (26), we have $\lim _{r \rightarrow \infty} h^{110}(r)=3 M^{2}$, a limiting behavior that is exactly fulfilled in our calculations by both simulated and theoretical results. In the figure, we have subtracted the corresponding limiting values of the correlation functions so that the plots present the same asymptotic limit of zero for the two temperatures, which facilitates their comparison. The increase in the range of the correlations with the lowering of the temperature is readily appreciated. The agreement between theory and simulation is remarkable in both cases.

To get an estimate of the critical inverse temperature from the orderedphase results, we fit the RZH magnetization in the vicinity of the critical temperature $(K<0.3)$ to a power law, $M=a\left(K-K_{c}^{u}\right)^{\beta}$, which leads to $a=1.694, \beta=0.397$, and $K_{c}^{u}=0.254$. The fitted value of $\beta$ is close to the value $\beta=0.387$ reported by Nijmeijer and Weis [4] and to the critical 
TABLE 1. Magnetic and thermodynamic properties of the Heisenberg spin fluid for $\kappa=1$ and $\rho \sigma^{3}=0.7$ calculated in the RZH approximation for $\beta \mu B_{0}=0$. The values of $\chi_{y y}$ for $K>K_{c}$ correspond to the last converged solution. The $\mathrm{MC}$ value in parentheses is a rough estimate since, due to the vicinity of the critical point, the results at this temperature are particularly sensitive to system size. $\chi^{*} \equiv \chi / \rho \beta \mu^{2}$.

\begin{tabular}{ccccccccc}
\hline$K$ & $\beta \mu B$ & \multicolumn{2}{c}{$\beta U / N$} & $\beta p / \rho$ & $\rho k_{B} T K_{T}$ & $\chi_{z z}^{*}$ & $\chi_{y y}^{*}$ & $\alpha$ \\
& & $\mathrm{RZH}$ & $\mathrm{MC}$ & & & & & \\
\hline 0.10 & 0 & -0.013 & & 5.685 & 0.0574 & 0.574 & 0.574 & 10.0 \\
0.15 & 0 & -0.034 & -0.034 & 5.668 & 0.0576 & 0.877 & 0.877 & 10.0 \\
0.20 & 0 & -0.074 & -0.073 & 5.635 & 0.0580 & 1.736 & 1.736 & 10.0 \\
0.25 & 0 & -0.156 & $-0.175)$ & 5.539 & 0.0601 & 8.854 & 8.854 & 4.00 \\
0.30 & 1.778 & -0.597 & -0.597 & 4.927 & 0.0618 & 1.655 & 8.534 & 1.88 \\
0.35 & 2.637 & -1.009 & -1.008 & 4.465 & 0.0657 & 0.666 & 7.733 & 1.92 \\
0.40 & 3.358 & -1.384 & -1.384 & 4.039 & 0.0694 & 0.366 & 6.312 & 1.90 \\
0.45 & 4.021 & -1.739 & -1.740 & 3.633 & 0.0734 & 0.245 & 5.424 & 1.86 \\
0.50 & 4.647 & -2.084 & -2.082 & 3.237 & 0.0779 & 0.209 & 5.841 & 1.81 \\
\hline
\end{tabular}

TABLE 2. Magnetic and thermodynamic properties of the Heisenberg spin fluid for $\kappa=1$ and $\rho \sigma^{3}=0.7$ calculated in the RZH approximation for $\beta \mu B_{0}=1 . \chi^{*} \equiv \chi / \rho \beta \mu^{2}$.

\begin{tabular}{|c|c|c|c|c|c|c|c|c|}
\hline \multirow[t]{2}{*}{$K$} & \multirow[t]{2}{*}{$\beta \mu B$} & \multicolumn{2}{|c|}{$\beta U / N$} & \multirow[t]{2}{*}{$\beta p / \rho$} & \multirow[t]{2}{*}{$\rho k_{B} T K_{T}$} & \multirow[t]{2}{*}{$\chi_{z z}^{*}$} & \multirow{2}{*}{$\chi_{y y}^{*}$} & \multirow[t]{2}{*}{$\alpha$} \\
\hline & & $\mathrm{RZH}$ & $\mathrm{MC}$ & & & & & \\
\hline 0.10 & 1.575 & -0.140 & & 5.539 & 0.0584 & 0.310 & 0.454 & 7.0 \\
\hline 0.15 & 2.018 & -0.297 & -0.298 & 5.365 & 0.0596 & 0.281 & 0.540 & 7.0 \\
\hline 0.20 & 2.548 & -0.517 & & 5.121 & 0.0615 & 0.227 & 0.618 & 7.0 \\
\hline 0.25 & 3.122 & -0.780 & -0.782 & 4.826 & 0.0638 & 0.174 & 0.679 & 7.0 \\
\hline 0.30 & 3.712 & -1.068 & & 4.505 & 0.0666 & 0.134 & 0.725 & 7.0 \\
\hline 0.35 & 4.304 & -1.367 & -1.365 & 4.170 & 0.0698 & 0.106 & 0.760 & 7.0 \\
\hline 0.40 & 4.896 & -1.673 & & 3.828 & 0.0734 & 0.088 & 0.786 & 7.0 \\
\hline 0.45 & 5.486 & -1.983 & & 3.482 & 0.0774 & 0.075 & 0.806 & 7.0 \\
\hline 0.50 & 6.073 & -2.295 & -2.295 & 3.133 & 0.0819 & 0.066 & 0.821 & 7.0 \\
\hline
\end{tabular}

exponent of the 3D lattice Heisenberg model, $\beta=0.362 \pm 0.004$ [23] and $\beta=0.3639 \pm 0.0035$ [24]. However, the available data from the integral equation magnetization are too far away from $K_{c}$ to draw here any conclusion with confidence and this agreement might simply be fortuitous. Nevertheless, a similar fit carried out on the second-order parameter $S$ produces the same estimate of $K_{c}^{u}$ and so we might conclude that this is the theoretical 


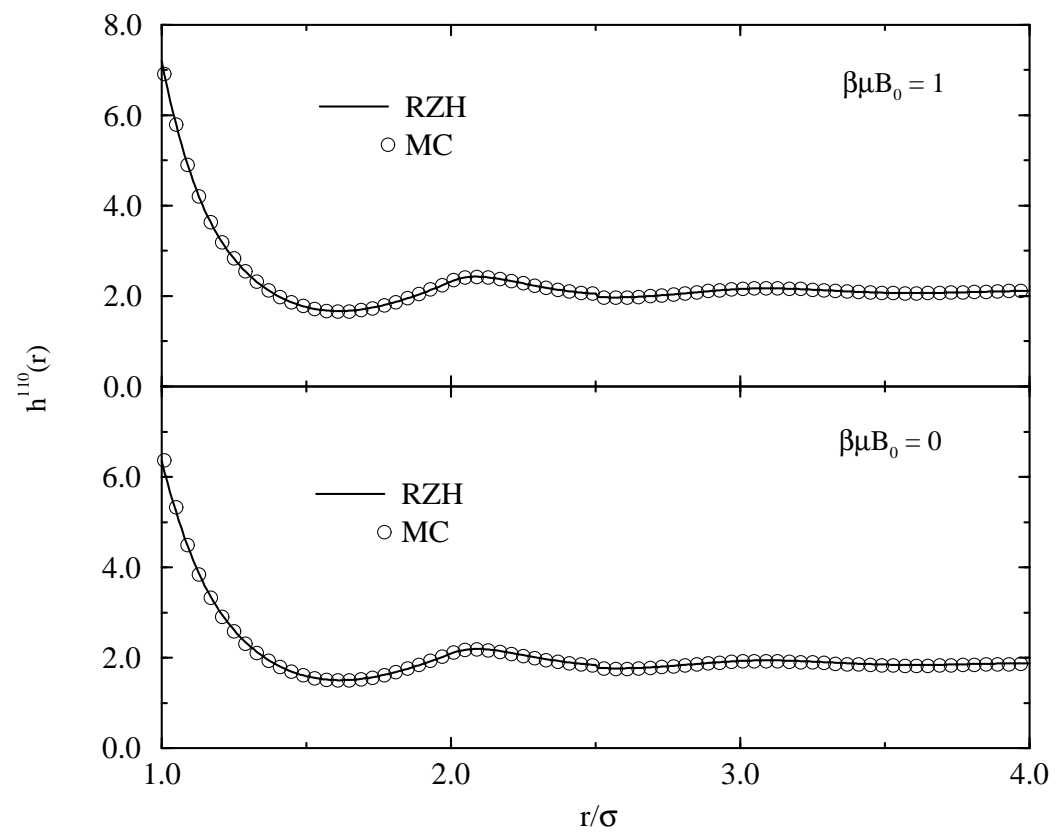

Figure 3. The $h^{110}(r)$ angular correlation function from MC simulation (white circles) and the RZH integral equation (solid lines) for $K=0.5$ and $\rho \sigma^{3}=0.7$ with and without an external magnetic field. The discontinuity at $r=2.5 \sigma$ is due to the potential truncation.

estimate of the critical inverse temperature derived from the ordered-phase data. The agreement between the critical point estimates $K_{c}^{l}=0.2645$ obtained in the disordered phase and $K_{c}^{u}=0.254$ obtained in the ordered phase can be regarded as good. Clearly, there is room for improvement, perhaps by imposing further consistency conditions. In particular, one would want $\chi_{z z}=\rho \mu \partial\langle x\rangle / \partial B_{0}$ to hold numerically.

The magnetic and thermodynamic properties of this set of states, including the effective field $B$, are summarized in Tables 1 and 2 for external fields $\beta \mu B_{0}=0$ and 1, respectively. Direct comparison with Monte Carlo data is available only for the internal energy $U$ and here we find excellent agreement with the results obtained using the RZH closure. That this excellent agreement between simulation and theory also extends to the correlation functions when the external field is turned on can be seen in Fig. 3. We note in Table 2 that for $\beta \mu B_{0}=1$ the consistency parameter $\alpha$ in the RZH closure is insensitive to changes in magnetic interaction strength; the determining factor is the hard sphere interaction. 


\section{References}

1. N.D. Mermin, Phys. Rev. A 137, 1441 (1965).

2. J.R. Henderson, in Fundamentals of Inhomogeneous Fluids (Dekker, New York, 1992), edited by D. Henderson, Chap. 2.

3. J. S. Høye and G. Stell, Phys. Rev. Lett. 36, 1569 (1976).

4. M. J. P. Nijmeijer and J. J. Weis, Phys. Rev. Lett. 75, 2887 (1995) ; Phys. Rev. E 53, 591 (1996).

5. J. J. Weis, M. J. P. Nijmeijer, J. M. Tavares, and M. M. Telo da Gama, Phys. Rev. E 55, 436 (1997).

6. E. Lomba, J. J. Weis, N. G. Almarza, F. Bresme, and G. Stell, Phys. Rev. E 49, 5169 (1994).

7. J. M. Tavares, M. M. Telo da Gama, P. I. C. Teixeira, J. J. Weis, and M. J. P. Nijmeijer, Phys. Rev. E 52, 1915 (1995).

8. See G. S. Cargill II and R. W. Cochrane, in Amorphous Magnetism, edited by H. O. Hooper and A. M. de Graaf (Plenum, New York, 1973), p. 313.

9. G. Bush and H. J. Guentherodt, Phys. Lett. 27A, 110 (1968); B. Kraeft and H. Alexander, Phys. Konden. Mater. 16, 281 (1973).

10. T. Albrecht, C. Bührer, M. Fähnle, K. Maier, D. Platzek, and J. Reske, Appl. Phys. A 65, 215 (1997).

11. J. P. Hansen and I. R. McDonald, Theory of Simple Liquids (Academic, London, 1986).

12. D. Henderson, in Fundamentals of Inhomogeneous Fluids (Dekker, New York, 1992), edited by D. Henderson, Chap. 4.

13. F. Lado and E. Lomba, Phys. Rev. Lett. 80, 3535 (1998).

14. F. Lado, E. Lomba, and J. J. Weis, Phys. Rev. E (to be published).

15. F. Lado, Phys. Rev. E 55, 426 (1997).

16. C. G. Gray and K. E. Gubbins, Theory of Molecular Fluids (Clarendon, Oxford, 1984), Volume 1.

17. See, for example, G. Arfken, Mathematical Methods for Physicists (Academic, Orlando, 1985), Chap. 9.

18. N. I. Akhiezer, The Classical Moment Problem (Hafner, New York, 1965), Chap. 1.

19. F. Lado, Mol. Phys. 47, 283 (1982).

20. Since the interatomic vector $\mathbf{r}_{12}$ is completely decoupled from the spin orientations $\hat{\mathbf{s}}_{1}$ and $\hat{\mathbf{s}}_{2}$ in the Heisenberg fluid, the solution of the $(\mathrm{OZ}+$ closure) equations here is actually simpler than that described in Ref. [19].

21. G. Zerah and J. P. Hansen, J. Chem. Phys. 84, 2336 (1986).

22. L. D. Landau, E. M. Lifshitz, and L. P. Pitaevskii, Electrodynamics of Continuous Media, (Pergamon, Oxford, 1984), Second Edition, Section 46.

23. C. Holm and W. Janke, Phys. Rev. B 48, 936 (1993).

24. K. Chen, A. M. Ferrenberg, and D. P. Landau, Phys. Rev. B 48, 3249 (1993). 